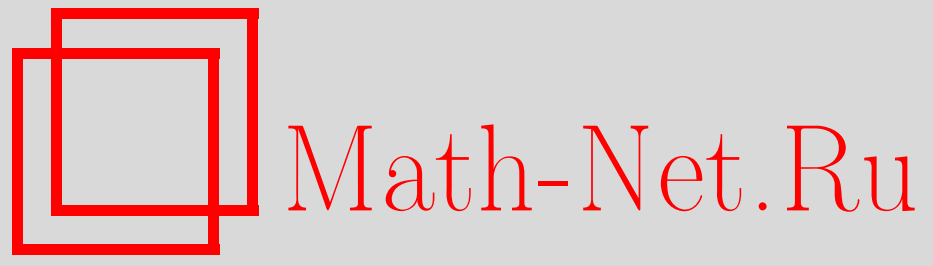

Обцероссийский математический портал

М. И. Нейман-заде, Эллиптические операторы с сингулярными коэффициентами, Матем. заметки, 2002, том 71, выпуск 5, 790-793

DOI: https://doi.org/10.4213/mzm653

Использование Общероссийского математического портала Math-Net.Ru подразумевает, что вы прочитали и согласны с пользовательским соглашением http://www.mathnet.ru/rus/agreement

Параметры загрузки:

IP: 54.224 .60 .19

26 апреля 2023 г., $17: 08: 46$ 


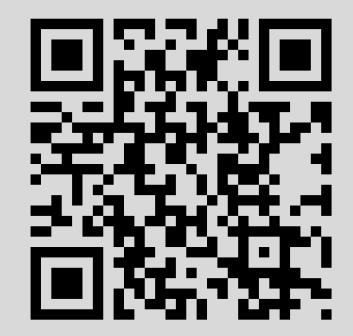




\section{ЭЛЛИПТИЧЕСКИЕ ОПЕРАТОРЫ С СИНГУЛЯРНЫМИ КОЭФФИЦИЕНТАМИ}

\section{М.И. Нейман-заде}

В этой заметке мы рассмотрим сильно эллиптические операторы вида

$$
L=\sum_{|\alpha|,|\beta| \leqslant m} D^{\alpha} c_{\alpha, \beta}(x) D^{\beta}
$$

в предположении, что $x \in \mathbb{R}^{n}$, а коэффициенты $c_{\alpha, \beta}$ - сингулярные функции. Точнее, мы будем предполагать, что коэффициенты главного символа (при $|\alpha|=|\beta|=m$ ) существенно ограниченные функции, а при $|\alpha|+|\beta|<2 m$ являюся обобщенными функциями. Основная цель работы найти достаточные условия на эти коэффициенты, гарантирующие корректность определения оператора $L$. Одновременно мы получим результаты о возможности приближения изучаемых операторов в смысле равномерной резольвентной сходимости операторами такого же вида, но с гладкими коэффициентами.

Решается также задача о приближении спектров и собственных векторов (если они имеются). Результаты заметки обобщают результаты работы [1], где аналогичные вопросы изучались для оператора Шрёдингера $L=-\Delta+q(x)$, в предположении, что $q(x)$ - обобщенная функция.

Пусть

$$
L_{0}=\sum_{|\alpha|=|\beta|=m} D^{\alpha} c_{\alpha, \beta}(x) D^{\beta}
$$

- главная часть оператора $L$. Мы используем стандартные обозначения

$$
D^{\alpha}=i^{|\alpha|} \frac{\partial^{|\alpha|}}{\partial x_{1}^{\alpha_{1}} \ldots \partial x_{n}^{\alpha_{n}}}, \quad \alpha=\left(\alpha_{1}, \ldots, \alpha_{n}\right) .
$$

С оператором $L_{0}$ ассоциируем полуторалинейную форму

$$
\mathscr{L}_{0}(u, v)=\int_{\mathbb{R}^{n}} \sum_{|\alpha|=|\beta|=m} c_{\alpha, \beta}(x) D^{\beta} u(x) \overline{D^{\alpha} v(x)} d x,
$$

определенную для функций $u, v \in S$, где $S$ - пространство Шварца гладких быстроубываюших функций. Далее предполагается, что функции $c_{\alpha, \beta}$ при $|\alpha|=|\beta|=m$ принадлежат пространству $L_{\infty}\left(\mathbb{R}^{n}\right)$, а также выполнена коэрцитивная оценка (неравенство Гординга)

$$
\operatorname{Re} \mathscr{L}_{0}(u, u) \geqslant \delta\left\|\nabla^{m} u\right\|_{L_{2}\left(\mathbb{R}^{n}\right)}^{2}, \quad u \in S,
$$

Работа выполнена при поддержке Российского фонда фундаментальных исследований, гранты № 01-01-00691 и № 00-15-96100. 
при некотором $\delta>0$. При условии $c_{\alpha, \beta}(x) \in L_{\infty}\left(\mathbb{R}^{n}\right)$ очевидна обратная оценка

$$
\left|\mathscr{L}_{0}(u, u)\right| \leqslant \text { const }\left\|\nabla^{m} u\right\|_{L_{2}\left(\mathbb{R}^{n}\right)}^{2}, \quad u \in S,
$$

а потому полуторалинейная форма $\mathscr{L}_{0}(u, v)$ является секториальнй $[2$, гл. 6]. Следовательно, эта форма замыкаема, и согласно первой теореме о представлении существует единственный оператор в пространстве $L_{2}\left(\mathbb{R}^{n}\right)$, который мы также обозначим через $L_{0}$, такой, что

$$
\mathscr{L}_{0}(u, v)=\left(L_{0} u, v\right)_{L_{2}\left(\mathbb{R}^{n}\right)}, \quad u, v \in S .
$$

Пусть $A_{0}$ - самосопряженный оператор, отвечающий (согласно первой теореме о представлении) положительной вещественной квадратичной форме $\operatorname{Re} \mathscr{L}_{0}(u, u)$. Из оценок $(3),(4)$ легко следует, что область определения замькания квадратичных форм $\operatorname{Re} \mathscr{L}_{0}(u, u)$ и $\mathscr{L}_{0}(u, u)$ совпадает с областью определения квадратного корня $A_{0}^{1 / 2}$ и совпадает с соболевским пространством $H^{m}\left(\mathbb{R}^{n}\right)$. При этом известно [2, гл. 6 , теорема 3.2$]$, что оператор $L_{0}$ допускает представление

$$
L_{0}=A_{0}^{1 / 2}(1+i B) A_{0}^{1 / 2},
$$

где $B$ - некоторый ограниченный оператор в $L_{2}\left(\mathbb{R}^{n}\right)$. В частности, оператор $L_{0}$ гомеоморфно переводит пространство $H^{m}$ в двойственное пространство $H^{-m}\left(\mathbb{R}^{n}\right)$.

Пусть $k, l \geqslant 0$. Обобщенную функцию $\varphi \in S^{\prime}$ назовем мультипликатором из $H^{k}\left(\mathbb{R}^{n}\right)$ в $H^{-l}\left(\mathbb{R}^{n}\right)$, если

$$
|(\varphi f, g)| \leqslant \text { const }\|f\|_{k}\|g\|_{l} \quad \forall f, g \in S,
$$

причем константа в неравенстве не зависит от $f$ и $g$. В этой записи $(\varphi f, g):=(\varphi, \bar{f} g)$ - значение функционала $\varphi f$ на функции $g$ из основного пространства $S$. Для $\varphi \in S^{\prime}$ и $f \in S$ определен оператор умножения $M_{\varphi} f=\varphi f$. Неравенство (6) эквивалентно тому, что $M_{\varphi}$ продолжается как ограниченный оператор из пространства $H^{k}\left(\mathbb{R}^{n}\right)$ в $H^{-l}\left(\mathbb{R}^{n}\right)$.

Далее множество $\varphi \in S^{\prime}$, для которого выполнена оценка (6), обозначаем через $M\left[H^{k} \rightarrow H^{-l}\right]$ или коротко $M[k,-l]$. Очевидно, линейное пространство $M[k,-l]$ превращается в банахово пространство, если норму в нем определить как инфинум констант, для которых выполнена оценка (6). В пространстве $M[k,-l]$ выделим подпространство $M_{0}[k,-l]$, состоящее из мультипликаторов $\varphi$ таких, что $\forall \varepsilon>0$ найдется константа $C(\varepsilon)$, гарантирующая оценку

$$
\|\varphi f\|_{-l} \leqslant \varepsilon\|f\|_{k}+C(\varepsilon)\|f\|_{-l} \quad \forall f \in S .
$$

Из определений легко следует, что $M[k,-l]=M[l,-k], M_{0}[k,-l]=M_{0}[l,-k]$.

Приведем основные результаты заметки.

ТЕОРема 1. Пусть $k$-челое число, $k \geqslant l u k>n / 2$. Тогда $H_{2}^{-l} \subset M_{0}[k,-l]$.

Tеорема 2. Пусть $k$ - иелое число, $k \geqslant l u k<n / 2$. Тогда $H_{\gamma}^{-l} \subset M_{0}[k,-l] n p u$ $\gamma \geqslant n / k$.

TEOPEMA 3. Ecлu $k \geqslant l u k<n / 2$, mo $H_{\gamma}^{0}=L_{\gamma}\left(\mathbb{R}^{n}\right) \subset M_{0}[k,-l] n p u \gamma \geqslant n /(k+l)$. Пpu $k=n / 2$ влохсение сохраняется, если $\gamma>n /(k+l)$.

Теорема 4. Пусть функиия $\varphi$ зависит от р переменных и принадлежит пространству $M[k,-l]$ в $\mathbb{R}^{p}$, а функиия $\psi$ зависит от $q$ переменных и принадлехит пространству $L_{\infty}\left(\mathbb{R}^{q}\right)$. Тогда функция $\varphi(x) \psi(y) \in M[k,-l]$ в $\mathbb{R}^{p+q}$ и справедлива оченка

$$
\|\varphi \psi\|_{M[k,-l]} \leqslant\|\varphi\|_{M[k,-l]}\|\psi\|_{L_{\infty}} .
$$

Ecли же $\varphi \in M_{0}[k,-l]$, mо $\varphi \psi \in M_{0}[k,-l]$ в $\mathbb{R}^{p+q}$. 
Tеорема 5. Пусть оператор L вида (1) таков, что коэффичиенты его главной части $L_{0}$ (имеющей представление (2)) являются существенно ограниченными и выполнена коэриитивная оченка (3). Пусть коэффичиенты в (1) таковы, что

$$
c_{\alpha, \beta}(x) \in M_{0}[m-|\alpha|,|\beta|-m] \quad n p u|\alpha|+|\beta|<2 m .
$$

Тогда полуторалинейная форма $(L u, v)$ (определенная при $u, v \in S$ ) продолжается по непрерывности как замкнутая секториальная форма с областью определения $H^{m}\left(\mathbb{R}^{n}\right)$. Тем самым, формальному дифференциальному выражсению (1) согласно первой теореме о представлении ставится в соответствие секториальный оператор в $L_{2}\left(\mathbb{R}^{n}\right)$, который продолхается по непрерывности как биективное отображение из $H^{m}\left(\mathbb{R}^{n}\right)$ в $H^{-m}\left(\mathbb{R}^{n}\right)$.

ЗАмечАниЕ 1. Если предположить, что $c_{\alpha, \beta}$ при $|\alpha|=|\beta|=m$ непрерывны, то коэрцитивная оценка (3) вьполняется автоматически для равномерно сильно эллиптических операторов $L_{0}$ (cм. [3]).

Теорема 6. Утверждение теоремы 5 имеет место, если для всех $\alpha, \beta$ таких, что $|\alpha|+|\beta|<2 m$,

$$
\begin{array}{ll}
c_{\alpha, \beta}(x) \in H_{\gamma}^{|\alpha|-m}\left(\mathbb{R}^{n}\right) & n p u|\alpha| \geqslant|\beta|, \gamma>\max \left(2, \frac{n}{m-|\beta|}\right), \\
c_{\alpha, \beta}(x) \in H_{\gamma}^{|\beta|-m}\left(\mathbb{R}^{n}\right) & n p u|\alpha| \leqslant|\beta|, \gamma>\max \left(2, \frac{n}{m-|\alpha|}\right) .
\end{array}
$$

В случае $m-|\alpha| \neq n / 2(m-|\beta| \neq n / 2)$ допускаются также значения $\gamma=\max (2, n /(m-|\beta|))$ $(\gamma=\max (2, n /(m-|\alpha|)))$.

Tеорема 7. Обозначим через $L_{n}$ операторы вида (1) с главной частью $L_{0}$ и гладкими коэффичиентами $c_{\alpha, \beta, n}(x)$ при $|\alpha|+|\beta|<2 m$. Предположим, что при $|\alpha| \geqslant|\beta|$ (nри $|\alpha| \leqslant|\beta|$ симметрично) последовательности функиий $c_{\alpha, \beta, n}$ сходятся $\kappa c_{\alpha, \beta}(x)$ в норме пространств $H_{\gamma}^{|\alpha|-m}\left(\mathbb{R}^{n}\right), \gamma \geqslant \max (2, n /(m-|\beta|))($ при $m-|\alpha|=n / 2$ полагаем $\gamma>2)$. Тогда последовательность операторов $L_{n}$ сходится $\kappa$ оператору $L$ в смысле равномерной резольвентной сходимости.

ЗАмЕч АниЕ 2 . В теореме 7 достаточно потребовать сходимости последовательностей $\mathrm{c}_{\alpha, \beta, n}(x)$ в норме пространств $M[m-|\alpha|,|\beta|-m]$.

ТЕОРема 8. Пусть выполнены условия теоремы 5 и оператор L, соответствующий дифференциальному выражению (1), имеет изолированное собственное значение $\lambda_{0}$ конечной алгебраической кратности р, причем длина максимальной жордановой чепочки равна $p_{0}$. Предположим, что последовательности гладких функиий $c_{\alpha, \beta, n}$ сходятся $\kappa$ $c_{\alpha, \beta}$ в норме пространств $M[m-|\alpha|,|\beta|-m]$. Тогда при достаточно больших $n$ найдутся $p$ собственных значений $\left\{\lambda_{n, k}\right\}_{k=1}^{p}$ соответствующ, операторов $L_{n}$, для которых выполняется оченка

$$
\left|\lambda_{n, k}-\lambda_{0}\right| \leqslant \mathrm{const}\left(\sum_{|\alpha|,|\beta|=m}\left\|c_{\alpha, \beta, n}(x)-c_{\alpha, \beta}(x)\right\|_{M[m-|\alpha|,|\beta|-m]}\right)^{1 /\left(2 p_{0}-1\right)} .
$$

Если $\lambda_{0}$ - простое собственное значение и ему соответствует собственная функиия $f$, то соответствующие собственнье функиии операторов $L_{n}$ сходятся $\kappa f$, причем

$$
\left\|f_{n}-f\right\|_{H^{m}} \leqslant \mathrm{const} \sum_{|\alpha|,|\beta|=m}\left\|c_{\alpha, \beta, n}(x)-c_{\alpha, \beta}(x)\right\|_{M[m-|\alpha|,|\beta|-m]} .
$$

Автор благодарит профессора А. А. Шкаликова за постановку задач и ценные советы. В частности, при доказательстве теорем 5 и 6 были использованы результаты Шкаликова и Бака о мультипликаторах в пространствах Соболева с негативными индексами гладкости. 


\section{СПИСОК ЦИТИРОВАННОЙ ЛИТЕРАТУРЫ}

1. Нейман-заде М. И., Шкаликов А. А. // Матем. заметки. 1999. Т. 66. № 5. С. 723-733. 2. Като Т. Теория возмущений линейных операторов. М.: Мир, 1972. 3. Йосида К. Функциональньй анализ. М.: Мир, 1967.

Московский государственный университет им. М. В. Ломоносова 